\title{
Operational constraints on dimension of space imply both spacetime and timespace
}

\author{
Jakub Czajko \\ Science / Mathematics Education Department \\ Southern University and A\&M College Baton Rouge, LA 70813, USA \\ E-mail address: sunswing77@netscape.net
}

\begin{abstract}
Since polynomials of higher than fourth degree, which is the algebraic counterpart of generic geometric dimension, are insolvable in general, then presumably no more than just four mutually orthogonal geometric dimensions can be placed within a single geometric space if it is expected to be fully operational. Hence a hierarchical notion of dimension is needed in order to ensure that at least virtual orthogonality is respected, which in turn implies presence of certain hierarchically organized multispatial structures. It is shown that the operational constraint on physical spaces implies of necessity presence of both: 4-dimensional (4D) spacetime and a certain 4D timespace.
\end{abstract}

Keywords: Insolvable polynomials; resolvents; higher dimensions; spacetime; timespace

\section{INTRODUCTION}

At elementary depth of inquiry into geometry, dimensionality usually was introduced by analogy. A point in n-dimensions was often defined as an ordered set of quantities and [allegedly] there is no real difficulty in making $\mathrm{n}$ as large as we please [1]. In the same manner as points of a plane and planar set, one could define points of $n$-dimensional (nD) space and abstract $\mathrm{nD}$ point-sets [2], which are identical with [polar] vectors [3]. This identification essentially equates the point-set space with Euclidean vector space, which is a [linear] vector space (LVS) endowed with scalar product of vectors [4].

Although the approach can be used to introduce higher-dimensional spaces, it creates by decree an artificial mathematical reality (or a world that exists only on paper), because such definitions are actually also existential postulates in disguise. For it tacitly equates mathematical foundations of our physical reality with a single geometric space and thus explictly postulates that there is neither operational nor conceptual limit on the number of generic dimensions of any single geometric space despite the (poven by Abel and then also by Galois) fact that polynomials of higher than 4th degree are insolvable in general.

Consequently thus, for matrices of degree $n>4$ their space of solutions represented by their characteristic polynomials, which can also be viewed as the most abstract (as being direction-independent) algebraic dimensions, cannot be always determined. If so, then in what sense may we still meaningfully speak of $\mathrm{nD}$ spaces for $\mathrm{n}>4$ and respect the abstract algebraic limit on dimensions of single space proven by Abel and enhanced by Galois?

The commonly used approach to pure mathemtics just ignores some conveniently forgotten, conceptually inconvenient but otherwise unquestionable, achievements of Abel, 
Galois, Lagrange, Dedekind and Hausdorff, to name just a few most prominent precursors of realistic trends in abstract and (destined to be) applied mathematics. Since group theory as a whole is accepted in mathematics and unreservedly applied, then why are some of its uncontrovertible consequences just tacitly disregarded in geometry?

I am not saying that all mathematical achivements of these thinkers were ignored, but only that their somewhat controversial and thus somehow inconvenient results are tacitly disregarded or overlooked at best. But now even string theories hit the proverbial wall of mathematical defiance of both physical and even its own mathematical reality with their discovery of strange behavior of certain duality pertinent to squeezing strings, which results in curious reexpansion of their stringy space into yet another dimension [5-7] p. 29. This behavior is unexplainable from the current conceptual standpoint of pure mathematics. Because mathematics keeps on ignoring the aforesaid controversial achievements (including those founded by Lagrange in reference to his resolvents, which behave similarly to the squeezed stringy space) I shall revisit those issues.

Notice that the reexpansion is not driven by any direct changes to distribution of a material substance filling the stringy space (or of the underlying it field), but seems to be a structural response to the abstract representation of the string that is squeezed within the space, which is apparently morphing itself in order to kindly accomodate new geometric representation of the process of squeezing. The effect of the procedural squeezing affects ennergy, which in turn somehow reshapes the space's structure that corresponds to the procedure and thus effectively must change the representation of quantized momentum - see [7] p. 29. Although the sqeezing can be fairly well described in narrative terms of physics, it remains unexplainable - neither by former physics nor former mathematics.

By theories of former mathematics and physics I mean those developed prior to 2000 $\mathrm{AD}$, when I demonstrated that radial/center-bound force fields can also have some clearly nonradial effects whose existence [8] has already been confirmed in few direct [9] and suggested by several indirect experiments [10-13]. For presence of nonradial effects of radial fields indicates inadequacy of several theories of previous mathematics and physics, which assumed that radial fields cannot produce any nonradial effects.

Pure mathematics used to consider various spaces and abstract manifolds defined over point-sets. Those abstract spaces/manifolds have internal structures established and organized by relations between their points. The reexpansion of stringy space and curious behavior of Lagrange resolvents (which also inexplicably increase the dimensionality of resolvents for higher than $4^{\text {th }}$ degree polynomials) indicate thus that - in addition to their internal structures - these abstract spatial structures apparently have also certain external structures, the totality of which can form multispatial hyperspaces (MH) [14,15].

This indicates, however, that in addition to mapping numbers/points, the manifolds containing them can also undergo some external morphing which may include change of base of linear vector space (LVS) associated with the point-set space, something that was not really considered by former pure mathematics thus far. Recall that the mathematical catastrophe theory, which was originally intended as a universal abstract morphogenesis, pertains primarily to internal morphing of shapes of objects immersed within the abstract spatial structure that is viewed as scaffolding of the generic space, but the structure itself was usually assumed as always remaining intact during the process of space's internal morphing. The curious behavior of Lagrange resolvents suggests that the spatial structure can be morphing too, and the reexpansion of stringy space reaffirms this supposition.

Although René Thom originally recognized only two types of distinct catastrophes, namely the catastrophe of conflict and that of bifurcation [16], he has also envisioned yet another catastrophe of transition through or near singularity, which could correspond to a certain compactification at infinity [17]. If the latter catastrophe would be treated just as 
transition between two single geometric spaces within the context of a certain spanning them abstract spatial structure, one could view it also as a kind of an external morfing, provided that the transition is enforced by an external (i.e. pangeometric) symmetry.

There is no doubt that presence of quasi-gemetric $\mathrm{nD}$ objects can be imagined even when $n>4$. What could be questioned, however, is whether such objects - and the abstract sets in which they would be immersed - can still be called 'spaces' and how the higher than 4th dimensions could be unambiguously distributed among such higher-dimensional objects and/or quasi-spatial structures. We need fairly precise (and capable of being fully operated on) notion of dimension which could be applied also in mathematical theories of physics. Acceptable notion of dimension should not cause cognitive conflicts which can prevent assimilation of some newly acquired controversial knowledge with - previously accepted as true - proven mathematical laws/rules. Decent mathematics should be able to handle any number of dimensions [18] without sacrificing its integrity though.

Unwilling to face tough conceptual challenges driven primarily by curious and thus unexplained experimental results, former pure mathematics embraced easier to handle settheoretical (and by proxy also group-theoretical and topological) approach to solving its own (and physical) problems.

It would be commendable were it not for the fact that it negelected conceptually inconvenient algebraic and geometrical issues. Because topology and group theory are founded upon incomplete set of axioms, they are richer but also their application domain is narrower [19]. Therefore they cannot carry on alone.

Unbiased mathematical proofs may be considered as thought experiments, provided they do not rely on any postulated existence of objects. Existential assertions regarding objects should arise either from valid operations of procedures or from constructions of structures. Note that representations (such as structures or procedures) are merely modes of existence of objects, not really objects.

Existence of objects that emerges from making procedures operational (or from proving that structures corresponding to those procedures are constructible) should be left to direct (or at least indirect) experimental confirmation.

It is because we will always encounter some new and presumably also few unanticipated aspects of reality, which can derail even best theoretical reasonings that were based upon those previously developed paradigms that did not take into account those new aspects. It is of extreme conceptual importance to realize that structural equations of (constructible) geometric objects actually involve certain procedural elements (such as projections - see [20] p. 187).

For if the procedures are not operationally feasible then the corresponding to them structures cannot be constructed, even if their equations are written down on paper. This is especially pronounced for geometric structures that are dynamic - compare [21]. 


\section{SYMMETRIES CONSTRAIN POLYNOMIALS AND DIMENSIONS}

Right-down sliding diagonal of an arbitrary $3 \times 3$ square matrix A of the generic form

$$
\left[\begin{array}{ccc}
\mathrm{a}_{11}-\lambda & \mathrm{a}_{12} & \mathrm{a}_{13} \\
\mathrm{a}_{21} & \mathrm{a}_{22}-\lambda & \mathrm{a}_{23} \\
\mathrm{a}_{31} & \mathrm{a}_{32} & \mathrm{a}_{33}-\lambda
\end{array}\right]=0
$$

yields a certain cubic equation in $\lambda$ called characteristic polynomial of the matrix [22]

$$
\left(\mathrm{a}_{11}-\lambda\right)\left(\mathrm{a}_{22}-\lambda\right)\left(\mathrm{a}_{33}-\lambda\right)=0
$$

and the function $f(\lambda)=\lambda I-A$ gives the characteristic matrix of the given matrix A [23] where $\mathrm{I}$ is an identity matrix. Evidently we can associate some matrix with every linear transformation of a finite-dimensional linear vector space (LVS) into another [24].

By Hamilton-Cayley theorem every square matrix A always satisfies its very own characteristic equation $|\mathrm{A}-\lambda \mathrm{I}|=0$ with the eigenvalues $\lambda$ taken from its diagonal $[25,26]$, [27]. Hence the degree of the characteristic polynomial corresponds to most basic algebraic (i.e. direction-independent) generic dimension of the matrix's solutions space.

If the characteristic polynomial of the matrix determines abstract dimensionality of the space of solutions represented by the given matrix then dimensionality of the space is restricted by the operations that are driven by certain operational symmetries external to the space itself. If so then perhaps also certain structural symmetries beyond control of its matrix representation should play a role in codefining the generic abstract dimensionality.

Halmos pointed out that existence of abstract algebraic structures has to be coupled with presence of corresponding to them operational procedures, both of which shall be mutually dependent [28]. In other words: Since to every such procedure corresponds an abstract structure and vice versa, and the procedure obviously can transcend the space in which the structure lives, then what is the structure that determines its dimensionality?

A computer algebra-aided symmetry approach to investigating integrability of some polynomial-nonlinear evolution equations in one temporal and just one spatial dimension is presented in [29]; the symmetry of spatial structures does not seem to emerge from it by extending dimensions so that another way to determine dimensions should be sought.

For there exist polynomials of degree greater than 4 with rational coefficients whose roots cannot be expressed via any combination of the rational numbers, the 4 arithmetic [number] field operations and the operation of integration [30]. An outline of proof that, in general, quintic equation could not be solved by any finite number of such algebraic operations has already been offered by Ruffini. In the past many great mathematicians, such as Bézout and Euler for instance, were inclined to believe that finding a solution to higherdegree polynomial equations was only a matter of some clever transformations. Note that most algebraists like Bézout used the term 'dimension' as just substitute for 'magnitude' or 'value' [31]. Even so, the algebraic dimensions represented by degrees of polynomials could be viewed as abstract precursors of generic geometric dimensions.

But the [attempted] Ruffini's proof of insolvability of polynomials of degree higher than 4 has reversed their opinion [32]. Thus it may seem that there is no reason to write any characteristic polynomials of $5^{\text {th }}$ degree or higher-dimensional matrices with all real coefficients, for what is the point in having equations that are insolvable? But this only means that the dream of modeling phenomena by writing a big $\mathrm{nD}$ polynomial of degree $\mathrm{n}>4$ with all dimensional variables written in one single line is just that: a dream. Hence one cannot fit 
more than 4 dimensions in just one single space, but the total number of dimensions that could appear in physical or mathematical reality is not really restricted.

Yet it also means that speaking of $n$-dimensional spaces (when $n>4$ ) without any additional qualifiers does not make sense either, for the mathematical operations are not made out of rubber to be expanded and twisted at one's convenience. Their rules must be respected. My point is that if no more than 4 dimensions could be fitted into any single space then perhaps we need to prepare more single spaces for any additional dimensions to be housed inside them, and if so then the extra spaces should be distinguished. This is the whole idea behind my conjectured existence of abstract structures called multispatial hyperspaces and their pangeometry (the theory to describe and handle them) $[14,15]$.

We also know today that a 5D matrix gives quite meaningful abstract representation of combined gravitational and electromagnetic equations. Nordström has extended 4D spacetime to 5D manifold in order to unify the electromagnetic and gravitational forces and the first tensorial 5D theory unifying these two forces has been proposed by Kaluza [33] p. 244. But since there is no evidence of those higher dimensions being present at shortest distances, then perhaps we should first develop a systematic method for studying the effects of the extra higher dimensions [34] before discussing Kaluza-Klein theory, which shall be done elsewhere. We need a new conceptual framework for that, however.

While not quite clear back then, the intellectual shock in geometry triggered by the fact of insolvability of higher than quartic equations was very troublesome indeed. The conceptual chaos caused by the shock continues till today, even though in the meantime mathematics has discovered all the pieces necessary to solve this cumbersome puzzle of dimensionality. However, the actual reason for the apparent inability to explain why the insolvability is really quite constructive (rather than destructive) conceptual constituent of the reality we live in, is our entrenched - though unwarranted - former paradigm of just single-space reality. The issue of general insolvability of higher than 4 degree polynomial equations suggests existence of multiple dual spatial structures overlying each other.

Logical necessity of the insolvability has eventually been proved by Abel [35-37], who also showed that some polynomials of degree 5 with real coefficients are not solvable by radicals [38-44]. Note that one can solve even an equation of degree 45 with conveniently chosen coefficients, for instance [45], but no general solutions for arbitrary polynomial equations of degree $\geq 5$ could ever be found. Although for certain particular unsolvable groups even a general solution can be obtained by using some differential invariants [46], no universally valid general solution exists for polynomial equations of degree $\geq 5$ [47], even though all arbitrary quartic polynomials are universally solvable by radicals. These facts have profound significance for geometry.

The Abel's result has been further advanced by Galois, whose theory supports both: general insolvability (in radicals) of polynomial equations of degree $\geq 5$ and solvability of polynomial equations of degree $\leq 4[48,49]$. The basic idea of Galois' theory is that by considering a class of Galois field extensions and determining all intermediate fields with the help of all subgroups of the abstract Galois symmetry group of the number field extension, the initially difficult (algebraic) field-theoretic problems can be reduced to much easier to solve (at least in abstract terms) group-theoretic problems [50]. This is in reference to algebraic fields (i.e. sets of real numbers equipped with the four most basic arithmetic operations: addition, subtraction, multiplication and division, provided the divisor is not equal to zero), which are both additive and multiplicative groups [51,52]. However, the fact that roots of polynomials can be expressed also by chain fractions [53] might still lead some to believe that algebraic dimensionality could grow inductively. In order to realize that this could not be a viable option, let us reconsider the Galois' result as a constructive assertion suggesting how 
higher than 3D objects could be established in actual reality, rather than as limitation posed on freedom of mathematical imagination.

The Galois' proof of insolvability of polynomials of degree $n>4$ together with the fact that no $4^{\text {th }}$ dimension placed within the $3 \mathrm{D}$ space could really be pairwise orthogonal to the other three geometric dimensions/directions is not a negative statement. It provides direct hint that the every $4^{\text {th }}$ dimension to be appended to any single 3D geometric space should reside within an extra single $3 \mathrm{D}$ space, yet the extra space has to be superimposed on the primary one and thus it cannot be standalone, but a dual space to the primary one.

The informal inductive introduction of $4^{\text {th }}$ dimension by generalization just cannot add even one more dimension to the 3D visual space [54]. In the sense the disregarded Galois' result virtually suggests necessity of presence of a certain abstract multispatial hyperspace without stating it explicitly. The MH should span at least two 4D dual spatial structures each of which should comprise two paired 3D single spaces [14,15]. At first glance this feat may seem impossible, but - unlike objects themselves - representations of objects are somewhat arbitrary and thus subject to interpretation. The validity of such representations is contingent only on correctness (of proper) operations to be performed on them, so that their structures (i.e. the structures that correspond to their actionable operational procedures) should be constructible. Their construction is up to mathematics but their existence is up to an experimental confirmation, preferably by physical means.

I shall show in this note that presence of an abstract multispatial hyperspace actually makes the insolvability logically necessary and therefore conceptually beneficial indeed. If phenomena seem weird, it is likely because the former mathematics previously used to describe them was conceptually too simplistic and often operationally wrong [10].

At this point I owe the reader also a clarifying note of caution: I am not saying that polynomials of degree $n>4$ are meaningless, but merely that their degrees do not really grow inductively even though they can surely grow incrementally. In other words: all the higherdimensional polynomials depict superimposed structures that appear somewhat hierarchical, not just as superposed additions to lower (n-1)D structures. As an example consider this: The fourth-order differential equation $\mathrm{P}\left(\mathrm{y}^{\prime}, \mathrm{y}^{\prime}, \mathrm{y}\right.$ "', $\mathrm{y}$ "') $)$ that Rubel has discovered is universal in the sense that any continuous function can be approximated with arbitrary accuracy over the whole $\mathrm{x}$-axis by a solution $\mathrm{y}(\mathrm{x})$ of the equation, which is a piecewise polynomial of degree 9 and of class $C^{4}$ [55]. Increasing dimensionality for $n>3$ is thus meaningful, but handling such abstract $\mathrm{nD}$ structures requires qualitatively different tools because the higher-dimensional structures are not just amorphous single point-set spaces, but compound hierarchical quasigeometrical spatial structures.

\section{LAGRANGE RESOLVENTS AND HIGHER DIMENSIONS}

Lagrange has alreday observed that that when the 4 roots of a quartic equation are permuted, the expression $r_{1} r_{2}+r_{3} r_{4}$ can assume only three [uniquely determined] values, namely itself and $r_{1} r_{3}+r_{2} r_{4}$ and $r_{1} r_{4}+r_{2} r_{3}$ [56]. He offered certain resolvents, which - as functions of roots of the polynomial equations - were supposed to reduce the degree of the original polynomial equation ([57] p. 178) in order to make it easier to solve. The Lagrange's method worked fine up to quartic equation, but for equations of $5^{\text {th }}$ degree the solution was ironiously "reduced" to much more complicated equation of a $6^{\text {th }}$ degree - compare $[58,59]$, [57] p.275. The fact that - otherwise meaningful - resolvents do not work above $4^{\text {th }}$ degree suggests that the procedural insolvability is not the result of an operational inefficiency, but it indicates that structural constructs restrict the procedures. 
Since the degree of a polynomial equation corresponds to the total number of abstract algebraic spatial extensions, hence it determines the admissible number of geometrically understood dimensions in a single number/point set (i.e. in an abstract algebraic space). It is about the total count of dimensions fitting a single space, not about their enumeration.

The number of such purely algebraic abstract dimensions corresponding to the degree of a polynomial equation is actually the count of degrees of freedom of the polynomial [60] $\mathrm{p}$. 74 , for from arithmetic of assumed algebraic of dimensions one could infer about the [total] number of such dimensions [61] involved therein. The degree may represent thus "pure and unadulterated" prototype of abstract dimension. Inductively progressing dimensions is an unwarranted demand rooted in philosophy of esthetics rather than in feasibility of operations. While philosophical ideas may serve as a guide, they must not impose unmathematical constraints that run contrary to operational/procedural and/or structural requirements of mathematics. Mathematics needs not be elegant or simple, but it should be true to our physical reality. The claim that pure mathematics creates abstract features more general than those existing in the reality we live in is not really always true if it ignores conceptually inconvenient operational restrictions on mathematical creations.

In fact, David Hestenes generalized this abstract idea onto spacetime algebra whose elements are called multivectors and can be expressed via polynomials over reals, each of which has at most 16 linearly independent elements [62]. Accidentally or not, we can see also 16 distributively generated near-rings [63]. But when dimensions of spacetime are defined by multivectors, the notion of dimension is virtually redefined from its original intuitive meaning that was rooted in geometry. Multivectors reveal yet another aspect of dimensionality visible mainly in gauge fields, but they did not really solve the riddle of curious behavior of Lagrange resolvents. Neither of these aspects is negligible.

Since solution of algebraic equation involves determination of its roots [64], if the roots cannot be determined for polynomial equations of degree higher than 4 , then the prospective paths described by such polynomials in spaces of solutions with more than 4 independently varying coordinate variables could not be uniquely determined within just one single space either. Hence no single 5D quasi-geometric generic space could ever be constructed, which implies that it could not exist outside one's confused mind. I am not saying that no abstract 5D quasi-spatial structure could actually exist, but only that such a structure is not, and could never be, a single geometric space like the 3D one with unique orthogonal dimensions. Hence calling the 5D structure 'space' can lead to tacitly veiled misconceptions or even nonsenses. While 5D abstract quasi-spatial structure can exists (i.e. it could be constructed within a multispatial framework), one needs new set of tools to handle it. While Galois has effectively proved impossibility of existence of 5D (and also higher-dimensional) quasi-spatial structures within the usual geometric paradigm of single-space reality, Lagrange virtually showed that unique representation of $\mathrm{nD}$ spatial structures requires - for $\mathrm{n}>4$ - superimposed pair of single spaces whose pairing involves mutually inverse morphing of one space into the other. Hence ignoring these two proven facts means admission of a fictional reality, which is an evasive response to these issues.

Although, at its face value, the fact that to simplify polynomial equation of $5^{\text {th }}$ degree one is required to upgrade it to (much more complicated) equation of $6^{\text {th }}$ degree might be disappointing, it is actually a valuable hint giving us otherwise unpredictable clue as to possible meaning of actual procedure for increasing the number of degrees of variability of orthogonal variables, and consequently also the number of quite independently varying abstract dimensions and distinct orthogonal coordinates (or generic directions), because by assigning coordinates to dimensions one could build $\mathrm{nD}$ projective spaces [65]. This is obviously yet another abstract operational restriction on the generic notion of dimension. 
Since generic orthogonality of a matrix [and of the transformation which the matrix represents] is defined as preservation of length [66], to require orthogonality is not really such a strange demand given the fact that elements of matrices are actually coordinates of vectors [67], [68], which have geometric interpretation in 3D space [69]. Hence in order for quasispatial structures with $n>3$ dimensions to preserve lengths and thus magnitudes of vectors, their dimensionality must be hierarchically split. For if orthogonality is a must and yet no more than 3 vectors could be orthogonal in any single geometric space, we should admit virtual orthogonality, which would indirectly impose orthogonality of base vectors via orthogonality of the spaces to which these bases belong. Orthogonality - as reflection of symmetry - is thus requirement of an operational law, and as such must not be compromised. But the way to implement the generalized virtual orthogonality is open.

In a sense, Galois has created an abstract dual theory to that of Lagrange [70]. The fact, however, that resolvents grow increasingly complicated when the total number of dimensions/degrees exceeds 4, virtually suggests that the essentially algebraic 4D spatial structures should be paired via their (aggregate) $4^{\text {th }}$ dimension. Moreover, the 3D single spaces of each pair should form such a 6D (algebraic) spatial structure that each of these two $3 \mathrm{D}$ single spaces is a dual image of the other. Since dual vectors are orthogonal [71], the duality pertains also to the two paired 3D single spaces. Notice that both group of $4 \mathrm{D}$ rotations and the Lorentz group $\mathrm{SO}(3,1)$ also have 6 independent parameters [72], which suggests that this conjectured abstract mapping of dual pairs of 4D spatial structures onto a single quasi-geometric 6D structure is truly universal fixture that should be considered as one of defining features of generic (i.e. whether geometric or algebraic) dimensions.

The two aforesaid conclusions also imply (again) that the linear vector spaces which are spanned over these two single 3D spaces should be somehow mutually symmetric and dual, yet with distinct and quite different dual bases. If so then presumably an inversion (from inside out) of the primary LVS (i.e. the single 3D space whose main representation is contravariant) into the secondary LVS (i.e. dual single 3D space whose representation is covariant with respect to the primary 3D space) should be somehow possible in order to facilitate the curious dimensional expansion of Lagrange resolvents, provided the two 3D spaces are contained within a 6D spatial structure. In effect this reasoning leads to a conjecture that a certain $8 \mathrm{D}$ hyperspace $(4 \mathrm{D}+4 \mathrm{D}=8 \mathrm{D})$ shall also exist beside the $6 \mathrm{D}$ one. Note that geometric duality replaces contravariance with covariance and vice versa [71].

I could have called the 8D spatial structure superspace, because these two 3D LVSs are superimposed on each other via a certain duality (whose actual character is yet to be determined), but the name 'superspace' has already been taken; it became associated with supersymmetry in physics. Thence at this point there is certain ambiguity, because there seem to exist two distinct kinds of hyperspaces (HS): $(3+3) \mathrm{D}$ HS which maps the primary 3D LVS onto its 3D dual LVS (resulting thus in unfurled 6D structure) as well as another one: $(4+4) \mathrm{D}$ HS which also maps one primary 4D abstract spatial structure onto its 4D dual counterpart (resulting thus in unfurled 8D structure, on a higher and quite different level of structural hierarchy though). Both of these abstract mappings are bi-directional though not quite identical - this distinction shall be explained in more detail elsewhere.

The two conceptual issues, namely: insolvability of polynomial equations of degrees $\geq 5$ (per Galois) and irreducibility of such equations to equations of lower degrees (per Lagrange) are the most fundamental operational hints suggesting presence of a certain higher abstract symmetry via which algebraic operations seemingly restrict prospective applicability of the generic notion of geometric dimension, but not their number. These two issues restrict the ways dimensions can be distributed in and among spaces. For not all dimensions of a solutions' space have to reside in the same, single geometric space. 


\section{GRASSMANN'S DIMENSIONS AS ALGEBRAIC EXTENSIONS}

Grassmann envisioned mathematics as a pure science of forms [73]. Within such an idealistic framework it seemed quite natural to combine the idea of algebraic extension with purely algebraic notion of polar vector represented by n-tuple of numbers [74,75]. He then postulated that so-designed magnitudes of vectors of higher extensions are to be obtained inductively via operation of outer multiplication [76]. From this point of view the geometric three-dimensional (3D) space appears as just a threefold extended number manifold with quadratic line element expressed by the sum of quadratic differentials of coordinates [77]. In order to identify the number manifold with Euclidean 3D space, however, an additional postulate of independent variability of each of these algebraic extensions, once they turn into abstract geometric dimensions, is definitely necessary.

However, independent variability implies orthogonality and thus also superposition of spatial effects. But the Grassmann's prescription for extending dimensionality can cause conceptual dilemma when a single 3D geometric space is to be extended to 4D spacetime. For in 4D spacetime the time-sense coordinate is superimposed on all three regular spatial coordinates rather than being just appended as an extra abstract algebraic extension to the 3D geometric space. For if one is going exactly in say the X-direction, for example, then this precludes one's going in either the Y- or the Z-direction of the 3D geometric space, but one must always go also in time [78]. In the sense the elapsing time (which is the only actively varying component of the fourth coordinate of 4D spacetime) always flows along all the three regular (i.e. purely spatial) directions and thus it should both be superposed and also superimposed on the three spatial (length-based) directions instead of being just superposed with them (i.e. the $4^{\text {th }}$ time-sense coordinate is not just appended to the three spatial coordinates). The three spatial directions are superposed, but not superimposed on each other. This subtle difference not only defeats the Grasmannian idea of dimensionality as algebraic extension, but also defies inductive dimensionality.

Generalized abstract $\mathrm{nD}$ Euclidean space $\mathbb{R}^{\mathrm{n}}$ is usually defined algebraically as the Cartesian product $\mathbb{R}^{n}=\mathbb{R} \times \ldots \times \mathbb{R}$ taken $n$ times, in which case one can view each onedimensonal subspace $\mathbb{R}$ as an additive group [79]. Evidently if an 1D time-based line is treated as just a subspace $\mathbb{T}$, it would be merely superposed on the regular length-based subspaces/lines $\mathbb{R}$ as $\mathbb{R}^{4}=\mathbb{R} \times \mathbb{R} \times \mathbb{R} \times \mathbb{T}$ in order to form the 4D quasi-spatial structure, then such combined $\mathbb{R}^{4}$ could not be identified with true $4 \mathrm{D}$ spacetime. Neither arbitrary purely algebraic (nor set-theoretic, nor topological, nor group-theoretical, nor geometric, nor any other purely mathematical) approach could supply - all by itself - conceptually viable definitions of 4D mathematical structures with relevance also to physical reality.

We need (new) synthetic mathematics that would take cues from objective - even if abstract - experiences, and from some (most likely unanticipated) curious experimental hints for that. It is not insinuation of a deficiency of pure mathematics, but an assertion that there should be a "separation of powers". For if any theory both defines its objects of interest without any external influence/control, and then also designs rules of operating on them, then it can quite inadvertently take (often quite unforeseable) logical shortcuts.

Notice that quadruple Cartesian product with fourth subspace/line $\mathbb{R}$ would not really create any realistic single 4D space, because as Elie Cartan remarked (in [20] p. 29) only if the quadratic form (of $\mathrm{n}$ contravariant coordinate components of the given vector) that represents metric/distance is [always] positive definite, it could be reduced to a sum of $n$ independent factors. Such an extra fourth dimension would not be orthogonal to the three regular ones without modifying the meaning of orthogonality that is tied to independent 
variability, both of which should remain unchanged if the mathematical framework of geometric dimension is not to become artificial (i.e. disassociated from the actual reality).

This fact does not make the notion of spacetime diagram any less significant than it is, but it suggests that 4D spacetime is an abstract spatial structure rather than geometrical space like the 3D geometric space of motion. This means lack of pairwise orthogonality, which would imply impossibility of separation of variables $[20,80,81]$ p. 29. If two variables cannot be separated, they must be somehow dependent on yet another variable.

Notice that, when defining n-dimensional structures in general, Poincaré did not call them spaces, but continua of $n$ dimensions redefined as sets of $n$ coordinates understood as $n$ directional quantities capable of varying independently of each other [82] p. 28.

Halmos wrote that constituents of an abstract mathematical structure should not just dangle separately, but must be subjected to approprite structural compatibility conditions; for a ring is not just a set in which both addition and multiplication can be performed - it is important that they be connected by the distributive law; and a topological group is not just a set that has a topological structure as well as multiplicative one - hence it is vitaly important that they be connected by continuity [83]. He also emphasized the other fact, namely that presumed existence of abstract structures has to be coupled with presence of certain operational procedures, both of which are mutually codependent [83].

For the fourth time-sense coordinate $\mathbb{T}$ of the 4D spacetime $\mathfrak{R}^{4}$ to be both superposed and superimposed on all three distances $\mathbb{R}$, we must demand different Cartesian product:

$$
\mathfrak{R}^{4}=\{\mathbb{R} \times \mathbb{R} \times \mathbb{R}\} \times \mathbb{T}^{1} \Rightarrow \mathfrak{R}^{4}:=\left\{\mathbb{R}^{3}\right\} \times \mathbb{T}^{1}
$$

which means that in length-based coordinates of the 4D spacetime $\mathfrak{R}^{4}$ the 4 th time-sense coordinate is $1 \mathrm{D}$ time line $\mathbb{T}^{1}$ whereas real distanes are intervals between $3 \mathrm{D}$ points $\left\{\mathbb{R}^{3}\right\}$

$$
\left\{\mathbb{R}^{3}\right\}:=\mathbb{R}_{\mathbb{R}}^{3}
$$

or represented by $3 \mathrm{D}$ vectors of the $3 \mathrm{D}$ LVS $\mathbb{R}_{\mathbb{R}}^{3}$ equipped with length-based vector-base.

The eq. (4) also implies that the spatial structure $\mathfrak{R}$ of $4 \mathrm{D}$ spacetime $\mathfrak{R}^{4}$ would have to possess tripartite time-sense coordinate in order to ensure that the 4 th dimension $\mathbb{T}^{1}$ (as the $1 \mathrm{D}$ time line pertinent to the elapsing time) - which, if undisturbed by motion - flows evenly, independently and simultaneously along all the other real spatial dimensions $\mathbb{R}$ as it is in the actual physical reality. Hence symmetry of duality of the spatial and temporal representations demands tripartite time-sense coordinate as a counterpart of 3D distances:

$$
\left\{\mathbb{R}^{3}\right\} \times \mathbb{T}^{1} \Rightarrow \mathbb{T}^{1}=\left\{\mathbb{T}^{3}\right\} \quad \text { or more precisely: } \quad \mathbb{R}_{\mathbb{R}}^{3} \times \mathbb{T}^{1} \Rightarrow \mathbb{T}_{\mathbb{T}}^{3} \times \mathbb{R}^{1}
$$

and consequently thus a dual 4D timespace $\mathfrak{T}^{4}$ should correspond to the 4D spacetime $\mathfrak{R}^{4}$

$$
\mathfrak{T}^{4}:=\left\{\mathbb{T}^{3}\right\} \times \mathbb{R}^{1}
$$

for existence of the 4D spacetime structure $\mathfrak{R}^{4}$ implies necessity of presence of dual 4D timespace $\mathfrak{T}^{4}$ with time-related base vectors though. This implication can be written as:

$$
\mathfrak{R}_{\mathbb{R}}^{4}=\mathbb{R}_{\mathbb{R}}^{3} \times \mathbb{T}_{\mathbb{R}}^{1} \Rightarrow \mathfrak{I}_{\mathbb{T}}^{4}=\mathbb{T}_{\mathbb{T}}^{3} \times \mathbb{R}_{\mathbb{T}}^{1}
$$

where both the spacetime and timespace are 4D quasi-spatial structures, not mere spaces. 


\section{TWO EQUIVALENT REPRESENTATIONS OF 4D SPATIAL STRUCTURES}

To arrive at the concept of 4D spatial structure by synthsizing its attributes it is clear that it should resemble a 4D abstract hyperspatial structure (HS), which should somehow comprise two quite distinct and mutually dual single 3D spaces, if the prospective objects depicted (if not immersed) within the HS have always to be solvable, at least in principle. Furthermore, by analogy to fibre bundle space (which also contains two distinct spaces: one of which is the 4D spacetime and the other is the gauge Lie's group space - both of which are connected so that every point of the spacetime is furnished with a copy of the gauge group space) [84], one could imagine a pair of two 3D heterogeneous dual spaces mutually coupled via a certain 4th dimension that would encapsulate the other 3D space.

By requiring hermicity of equations of motion operator as well as irreducibility with respect to Lorentz group representation one can prove that, in general, only metrics with signature corresponding to q time and (d-q) space dimensions - with q being odd - exist; hence some authors claim that in 4D space, the Nature could only make the realization of $(1+3) \mathrm{D}$ space [85]. However, the requirement of duality between spatial and temporal representations suggests that both $(1+3) \mathrm{D}$ and $(3+1) \mathrm{D}$ structures should be admissible.

Dedekind has already realized that although one needs 6 distinct equations for just 3 orthogonal (hence formally quite independent) lines, this seems to add only one extra requirement in two possible flavors, and as such makes dimensionality a pair of $1+3$ or $3+1$ kind [86]. Now there is also convincing proof, for it proceeds by contradiction, that there must be at least two 4D Euclidean spaces- see [87] for a very concise, informal (without mathematical formalism) review of more recent work on this and related issues.

Also Hausdorff admitted that it is actually impossible to distinguish between space and its perfect abstract mirror image [hence dual, in general] by some inner (geometric) criteria so that the two $(1+3) \mathrm{D}$ and $(3+1) \mathrm{D}$ seemingly equivalent (at least operationally) representations may be tied together in a way that topological methods did not recognize [88]. Note that this is not really a fault of topology. This issue is due mainly to the former paradigm of singlespace reality. No contemporary theory of most advanced mathematics or physics could even properly address the issue, not to mention grasping it. That is why (new) synthetic approach to mathematics (and physical sciences) is indispensable indeed.

However, in order to synthesize new concepts (or perhaps just enhancements to some already well-established notions) from curious experimental hints, it is required that one should renounce making any existential postulates, regardless of whether they are made explicitly or are disguised as definitions, for postulating existence can create nonexistent artificial reality and so it would defeat the very purpose of making prospective syntheses.

Although only 3 geometric dimensions can be mutually orthogonal within any single truly geometric space, one needs at least 4 dimensions to uniquely determine all possible configurations of the generic $3 \mathrm{D}$ objects immersed therein. It is known that characteristic polynomial does not uniquely determine the topology of a molecule - see [89] where an example of a pair of nonisomorphic connected graphs with the very same characteristic polynomial is given. Evidently Nature confirms that there is more than one structure of the ordinary 4D spacetime. Like it or not, but the physical reality is clearly multispatial. In order for mathematics to be relevant to physics, it has to enhance its old paradigm of single-space reality by admitting also presence of an abstract hierarchical multispatial hyperspace and explore the synthetic pangeometry to handle such multispatial structures.

The conclusion that 4D spacetime should resemble Cartesian product of some two 3D single spaces - as implied by the right-hand side (RHS) of the chain of equations (5) - is thus compatible with several theoretical as well as few experimental hints. Nonetheless, the unfurled time-sense term $\mathbb{T}^{3}$ does not really represent 3D flow of elapsing time, but merely 
1D curvilinear time line cast in three dimensions of superimposed single 3D space comprised within the conjectured abstract multispatial structure of regular 4D spacetime.

By stating that dimension of a vector space $\mathrm{V}$ is the maximum number of independent elements in V [90], pure mathematics trivialized the concept. We should not decide what dimension "is" before we realize how it can coexist in Nature with other related concepts and how it could be operated on. If treated as science, mathematics should discover the meaning of its concepts, structures and operational procedures, rather than to define them arbitrarily, because such man-made definitions are existential postulates in disguise.

Artlike/postulative mathematics often breeds conceptual nonsense, which - when it is applied to physical sciences - creates cognitive conflicts preventing assimilation of newly learned knowledge with some previously acquired, proven (and thus already accepted as true) knowledge in students and confusion among researchers. Postulates can defy reality.

\section{CONCLUSIONS}

Operational restrictions constraining generic dimensionality of physical spaces imply presence of both 4D spacetime as well as a certain 4D timespace that is dual counterpart of the spacetime. Since structural equations of objects immersed in $\mathrm{nD}$ spatial structures with $\mathrm{n}>$ 4 are insolvable in general, it is reasonable to cast such equations in a multispatial hyperspace comprising more than one solvable spatial structure. In other words: abstract multidimensionality for $\mathrm{n}>4$ require multispatiality to be implemented meaningfully.

The fact that the feasibility of both operations and construction effectively codefine geometric structures suggests that we should replace the traditional postulative approach to doing mathematics with an inquisitive one, which would synthesize new mathematical and physical concepts (and build up theories) from hints supplied by curious experiments.

\section{References}

[1] Kendall M.G., A course in the geometry of $n$ dimensions. New York: Hafner Publishing, 1961, p. 2.

[2] Brouwer L.E.J., Begründung der Mengenlehre unabhängig vom logischen Satz vom ausgeschlossenen Dritten II. Amsterdam, 1919, p. 3f.

[3] Hummel J.A., Vector geometry. Reading, MA: Addison-Wesley, 1965, p. 95.

[4] Audin M., Geometry. Berlin: Springer, 2003, p. 7.

[5] Schwarz J.H., Spacetime duality in string theory. [pp. 69-87 in: Schwarz J.H. (Ed.) Elementary particles and the universe. Cambridge: Cambridge Univ. Press, 1991].

[6] Witten E., Lectures on Quantum Field Theory. [pp.1225-1424 in: Deligne P. et al. Quantum Fields and Strings: A Course for Mathematicians II. Providence, RI: Am. Math. Soc., 1999, pp.1225ff.

[7] Witten E., Phys. Today April 1996, 24-30.

[8] Czajko J., Chaos Solit. Fract. 20 (2004) 683-700.

[9] Czajko J., Chaos, Solit. Fract. 11 (2000) 2001-2016.

[10] Czajko J., Stud. Math. Sci. 7(2) (2013) 25-39. 
[11] Czajko J., Stud. Ma th. Sci. 7(2) (2013) 40-54.

[12] Czajko J., International Letters of Chemistry, Physics and Astronomy 11(2) (2014) 89-105.

[13] Czajko J., International Letters of Chemistry, Physics and Astronomy 13(1) (2014) 32-41.

[14] Czajko J., Chaos, Solit. Fract. 12 (2001) 951-967.

[15] Czajko J., Chaos, Solit. Fract. 19 (2004) 479-502.

[16] Thom R. Modelès mathématiques de la morphogenèse. Paris: Christian Burgois Éditeur, 1980, p. 91.

[17] Thom R., Quid des stratifications canoniques. [p.375-381 in: Brasselet J.-P. (Ed.) Singularities. Lille 1991. Cambridge: Cambridge Univ. Press, 1994, see p. 376].

[18] Toth G., Glimpses of algebra and geometry. New York: Springer, 1998, p. 284.

[19] Efimov N.W., Höhere Geometrie. Berlin: VEB Deutscher Verlag der Wissenschaften, 1960, p. 224.

[20] Cartan E., Leçons sur la géométrie des espaces de Riemann. Paris: Gauthier-Villars, 1963, pp. 11, 14ff.

[21] Cartan E., Théorie des groupes finis et continus et la géométrie différentielle. Traitées par la méthode du repère mobile. Paris: Gauthier-Villars, 1937, p. 9ff.

[22] Kaplan W., Elements of ordinary differential equations. Reading, MA: Addison-Wesley, 1964, p. 169.

[23] Frazer R.A., Duncan W.J. \& Collar A.R., Elementary matrices and some applications to dynamics and differential equations. Cambridge: Cambridge Univ. Press, 1960, p. 64.

[24] Schneider H. \& Barker D.P., Matrices and linear algebra. New York: Dover, 1989, p. 217.

[25] Pettofrezzo A.J., Matrices and transformations. Englewood Cliffs, NJ: Prentice-Hall, 1966, p. 97.

[26] Thrall R.M. \& Tornheim L., Vector spaces and matrices. New York: Wiley, 1963, p. 257.

[27] Stewart F.M., Introduction to linear algebra. Princeton, NJ: Van Nostrand, 1963, p. 215.

[28] Halmos P.R., Austr. Math. Soc. 25 (1982) 161, see p.166.

[29] Gerdt V.P., Int. J. Mod. Phys. C 4(2) (1993) 279-286.

[30] Swallow J. Exploratory Galois theory. Cambridge: Cambridge Univ. Press, 2004, p.171.

[31] Bézout E., General theory of algebraic equations. Princeton, NJ: Princeton Univ. Press, 2006, p.7ff.

[32] Tignol J.-P., Galois' theory of algebraic equations. New York: Longman, 1988, p.274.

[33] Jammer M., Concepts of space. The history of theories of space in physics. New York: Dover, 1993, pp. 154, 173, 177. 
[34] Mansouri F., Witten L., Can isometries tell us about the extra dimensions? pp.509-511 [in: Barden W.A., White A.R. (Eds.) Symposium on anomalies, geometry, topology. Singapore: World Scientific, 1985].

[35] Gullberg J., Mathematics. From the birth of numbers. New York: W.W. Norton, 1996, p. 300.

[36] Weisstein E.W., CRC concise encyclopedia of mathematics. Boca Raton, FL: Chapman \& Hall/CRC, 2003, p.17.

[37] Cooke R., Classical algebra. Its nature, origins and uses. Hoboken, NJ: Wiley, 2008, p.118f.

[38] Abel N.H., A demonstration of the impossibility of the algebraic resolution of general equations whose degree exceeds four. [pp.271-6 in Stahl S., Introductory modern algebra. A historical approach. New York: Wiley Interscience, 1997.]

[39] Fraleigh J.B., A first course in abstract algebra. Reading, MA: Addison-Wesley, 1982, pp. $437 f f$.

[40] Cox D.A., Galois theory. Hoboken, NJ: Wiley, 2004, p. 200.

[41] Dobbs D. \& Hanks R., A modern course on the theory of equations. Washington, NJ: Polygonal Publishing, 1992, p.106.

[42] Houzel C., The work of Niels Henrik Abel. [pp.21-177 in: Laudal, O.A. \& Piene, R. (Eds.) The legacy of Niels Henrik Abel. The Abel bicentennial, Oslo, 2002. Berlin: Springer, 2004, p.50].

[43] Strang G., Linear algebra and its applications. San Diego: Harcourt Brace Jovanovich, Publishers, 1988, p. 251.

[44] Joyner D., Adventures in group theory. Baltimore: The Johns Hopkins Univ. Press, 2002 , p. 72.

[45] Livio M., The equation that couldn't be solved. New York: Simon \& Schuster, 2005, p. 285.

[46] Halburd R., Nonlinearity 12 (1999) 931-938.

[47] Serret J.-A., Cours d'algèbre supérieure 2. Paris, 1928, p. 512.

[48] Postnikov M.M., Fundamentals of Galois' theory. Delhi: Hindustan Publishing, 1961, p. 137.

[49] Nicholson W.K., Introduction to abstract algebra. New York: Wiley, 1999, pp.79, 512 \& 111 .

[50] Zeidler E. (Ed.) Oxford user's guide to mathematics. Oxford: Oxford Univ. Press, 2004, p. 676.

[51] Pontrjagin L., Topological groups. Princeton: Princeton Univ. Press, 1946, p. 171f.

[52] Hungerford T.W., Algebra. New York: Springer, 1974, p. 116.

[53] Lagrange J.L., Zusätze zu Eulers Elemente der Algebra. Unbestimmte Analysis. Leipzig, 1898, p. 81.

[54] Poincaré H., The value of science. New York: Dover, 1958, p.54.

[55] Duffin R.J., PNAS USA 78 (1981) 4661-4662. 
[56] Stahl S., Introductory modern algebra. A historic approach. New York: Wiley, 1997, p. 124.

[57] Netto E., The theory of substitutions and its applications to algebra. Bronx, MY: Chelsea, 1964, pp. 178, 275.

[58] Infeld L., Whom the Gods love. The story of Evariste Galois. New York: Whittlesey House, 1948, p. 58.

[59] Weber H., Lehrbuch der Algebra I. New York: Chelsea, p. 670.

[60] Marcus M. \& Minc H., Introduction to linear algebra. New York: Macmillan, 1969, p. 74.

[61] Hurewicz W., J. reine angew. Math. 169 (1933) 71.

[62] Hestenes D., J. Math. Phys. 16(3) (1975) 556-572 see p. 557.

[63] Malone J.J., Proc. Edinb. Math. Soc. 18 (1973) 235 see p.238.

[64] Uspensky J.V., Theory of equations. New York: McGraw-Hill, 1948, p. 82.

[65] Yano K., Les espaces a connexion projective et la géométrie projective des «paths ». [pp. 1-69 in: Obato M. (Ed.) Selected papers of Kentaro Yano. Amsterdam: NorthHolland, 1982, p. 10].

[66] Perlis S., Theory of matrices. New York: Dover, 1991, p. 179.

[67] Franklin J.N., Matrix theory. Mineola, NY: Dover, 2000, p. 75.

[68] MacDuffee C.C., Vectors and matrices. Menasha, WI: The Math. Assoc. of America, 1949 , p. 23.

[69] Tetra B.C., Basic linear algebra. New York: Harper \& Row, 1971, p. 75.

[70] Dehn E., Algebraic equations. An introduction to the theories of Lagrange and Galois. New York: Columbia Univ. Press, 1938, p.155.

[71] Mal'cev A.I., Foundations of linear algebra. San Francisco: W.H. Freeman, 1963, p. 276.

[72] Hladik J., Spinors in physics. New York: Springer-Verlag, 1999, p.135ff.

[73] Grassmann H., Gesammlte mathematische und physikalische Abhandlungen I/I: Die Ausdehnunglehre von 1844 und die geometrische Analyse. New York: Johnson Reprint Corp., 1972, p. 13ff.

[74] Kline M., Mathematical thought from ancient to modern times II. New York: Oxford Univ. Press, 1990, p. 784.

[75] Pettofrezzo A.J., Vectors and their applications. Englewood Cliffs, NJ: Prentice-Hall, 1966, p. 70.

[76] Grassmann H., Die Ausdehnungslehre von 1844 oder die lineale Ausdehnungslehre. Leipzig, 1878, p. 73.

[77] Lorenz K. (Ed.) Konstruktionen versus Positionen. Beiträge zur Diskussion um die Konstruktive Wissenschaftstheorie. Berlin: Walter de Gruyter, 1979, p. 495.

[78] Czajko J., Chaos Solit. Fract. 13 (2002) 17-23.

[79] Lang S., Algebraic structures. Reading, MA: Addison-Weslley, 1967, p.13.

[80] Da Costa R.C.T., Eur. J. Phys. 7 (1986) 269-273. 
[81] Da Costa R.C.T., Phys. Rev. A 25 (1982) 2893-2900.

[82] Poincaré H., Mathematics and science. Last essays. New York: Dover, 1963, pp. 17, 28.

[83] Halmos P.R., Austr. Math. Soc. 25 (1982) 161, see p. 166.

[84] Konopleva N.P., Kaluza-Klein or fibre bundles? [p.248-251 in: Barut A.O. et al. (Eds.) Quantum systems. New trends and methods. Singapore: World Scientific, 1995, see p. 249].

[85] Borštnik N. M., Nielsen H.B., Phys. Lett. B486 (2000) 314-321.

[86] Dedekind, R., J. reine angew. Math. 50 (1855) 272.

[87] Kolata G., Science 217 (1982) 432-433.

[88] Hausdorff F., Zwischen Chaos und Kosmos oder vom Ende der Mataphysik. BadenBaden: Agis Verlag, 1976, p.103.

[89] Balaban A.T., Harary F., J. Chem. Docum. 11 (1971) 258.

[90] Artin E., Galois theory. Notre Dame, IN: 1959, p. 4. 